\title{
Efficient Energy Clustering Protocol UsingGenetic Algorithm in Wireless Sensor Networks
}

\author{
Nandoori Srikanth* and Muktyala Siva Ganga Prasad \\ Department of Electronics and Communication Engineering, Kaneru Lakshmiah Educational Foundation, India
}

Received 19 October 2018; Accepted 29 December 2018

\begin{abstract}
Nowadays, Wireless Sensor Networks (WSNs) are pondered as an exploration subject. Currently, progress in electronic communications has directed to multi-purpose Sensor Nodes (SNs) with less price and power consumption. Energy efficiency is a major concern in WSNs as the sensor nodes are battery-operated devices. Clustering based techniques are implemented through data aggregation to make equal energy consumption among SNs for energy efficient data transmission. The existing clustering techniques make use of distinct Harmony Search Algorithm (HSA), Low-Energy Adaptive Clustering Hierarchy (LEACH) and Particle Swarm Optimization (PSO) algorithms. However, these algorithms have exploration exploitation trade-off and local search constraint individually. In order to obtain a global search with faster convergence, Efficient Energy Clustering Protocol (EECP) based on Genetic Algorithm (GA) is recently proposed to detect their immediate neighbors, balance energy consumption load among data transmission routes and energy efficient cluster head selection. The proposed algorithm exhibited high search efficiency and dynamic capability that improves lifetime of SNs. The presentation of the proposed algorithm was assessed using throughput, packet delivery ratio, energy consumption and end to end delay. The proposed algorithm showed an improvement in energy consumption and throughput by 95 and 90 Mbps respectively than existing clustering algorithm.
\end{abstract}

Keywords: Wireless Sensor Networks (WSN), Efficient Energy Clustering Protocol (EECP), Base Station (BS), Genetic Algorithm (GA).

\section{Introduction}

Sensor technologies and wireless communication in recent years have made wonderful evolution. Wireless Sensor Networks (WSNs) has used in an extensive diversity of utilizations and have appeared as an auspicious study field [1]. They have been utilized in environmental [2], smart home-field [3] and health field [4]. Complex environmental or physical circumstances can be monitored by using sensors in WSNs. Such sensors are normally furnished with the communication competences and data processing in order to assemble data and to track information back to Base Station (BS) [5]. Power source includes a limited energy budget battery which outcomes in determinate nodes lifetime in WSN. Moreover, it might be improbable or difficult for recharging the battery because nodes may be organized in an aggressive or impracticable environment [6]. The characteristic WSN scenario is given in Fig. 1.

Scholars are deeply intricate in energy efficient solutions planning, in the same way energy efficient network methods are the important parameters for improving network lifetime. The distributed WSNs saves energy by using Cluster based Hierarchical approach [7], which improves life of network by successfully using the node energy and supports lively environment of WSNs. Sensor nodes (SNs) in WSN are cluster based which is separated into numerous sets recognized as clusters with a group leader identified as Cluster Head $(\mathrm{CH})$. All sensor nodes sense data and propel it to reliable $\mathrm{CH}$ which lastly sends to the BS for extra processing. Clustering has many important gains over classical systems [8]. First, data aggregation is applied on data received from numerous $\mathrm{SNs}$ Within a cluster; to decrease the quantity of data transmitted to BS, consequently energy necessities reduces abruptly. Secondly, spin of $\mathrm{CHs}$ assistances to ensure balanced energy consumption within the network, which prevents receiving particular nodes famished because of absence of energy [9].

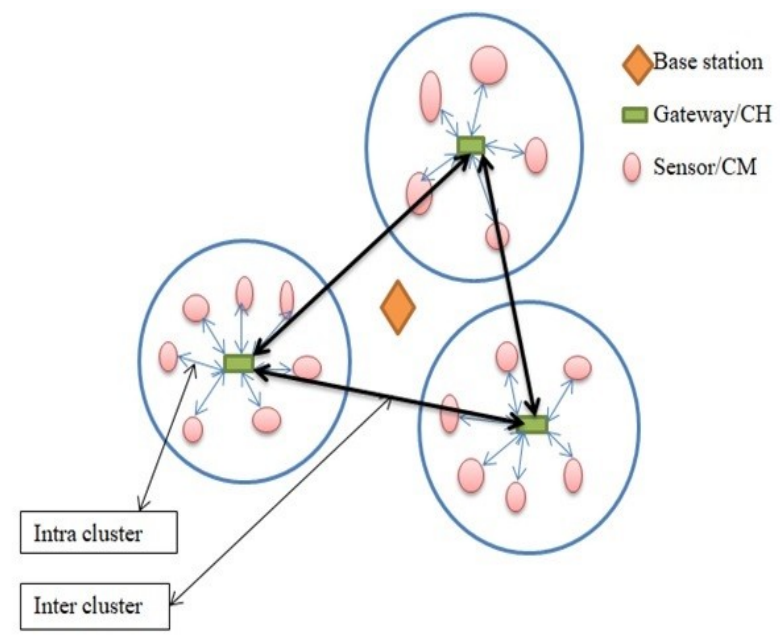

Fig. 1. Intra-cluster and Inter-cluster communication in WSN. 
Following considerations of clustering which decreases the number of broadcasts to prolong the network life time. A routing protocol was proposed to decrease redundant transmission towards the BS in utilization base heterogeneous WSN. Similar applications of BS nodes form sub-clusters and from these sub-clusters only one $\mathrm{SN}$ transmits data and the remaining SNs will be in sleep mode to save energy. The revolving epoch and election probability is correlated with the residual energy. Only high energy SNs belongs to a region have superior probability to become CHs. The $\mathrm{CH}$ also evades the similar data comes from dissimilar nodes move towards the BS. Like this, the redundant data is evaded which decreases the energy consumption. All the nodes are not constantly monitors the sensing field; only one awake node from a sub-cluster monitors the field in assigned time slots. The rest of the nodes were sleep mode to save theresources.

In this paper, a novel centralized Energy-efficient clustering protocol was proposed based on genetic algorithm in WSNs (named EECP-GA). In the clusterbased protocols from the literature, $\mathrm{CHs}$ are characteristically elected amongst all SNs and clusters are formed by simply allocating each node to the nearest $\mathrm{CH}$. However, EECP-GA general clustering is initially performed on all nodes to form balanced clusters and proper $\mathrm{CHs}$ are designated. The main contributions in this paper can be summarized as givenbelow:

- An optimized Genetic clustering algorithm was proposed as an efficient application specific routing protocol in WSNs.

- EECP-Genetic algorithm is used to form balanced clusters over the network.

- Genetic algorithm (GA) inference scheme is utilized to elect the suitable $\mathrm{CHs}$ and form clusters

- An artificial EECP algorithm is used to optimize the genetic algorithm rules to prolong the network lifetime, based on the application specifications.

- These Genetic algorithm rules should be adaptively tuned to prolong the network lifetime, once earlier EECP-GA operates for each utilization.

- An efficient energy clustering scheme was presented to denote feasible solutions and design a WSNs function to optimize the Genetic algorithm rules of EECP-GA.

The present study, aimed to reduce energy consumption by efficient energy clustering protocol based on genetic algorithms which had been proposed in recent years and leads to a rise in network lifetime are assessed. Finally, some advantages of GAs when compared to conventional methods are summarized and listed below.

\section{Advantages of Genetic Algorithm}

- Efficiency, reliability, parallelism, easily adapted for unlike glitches.

- Huge and wide solution space searching ability.

- Non-knowledge based optimization process.

- Use of fitness function forevaluation.

- Easy to evade trapping in local optima, discover global optimum.

- Capable of multi-objective optimization and can recurrence a suite of potential solutions.

- Respectable choice for large-scale/wide variety optimization glitches.

\section{Related Works}

Energy efficient and Quality of Service (QOS) aware routing protocol for Wireless Sensor and Actuator Networks (WSANs) delivers energy consumption and less delay. $\mathrm{CH}$ selected by accessibility degree in multi-hop distance of SNs concerning all the actuator nodes [10]. Though, the choice of suitable $\mathrm{CH}$ with ideal competences whereas network's complementary energy efficiency ratio is a distinct WSN's NP hard optimization problem [11]. Thus, Computational Intelligence (CI) [12] based approaches such as Artificial Immune System (AIS), Evolutionary Algorithms (EAs), Reinforcement Learning (RL) and added newly, Artificial Bee Colony (ABC) has been widely used as the population based metaheuristic for EECP in WSNs [13]. The protocol which associates energy efficient routing for specific applications with data aggregation and attains improved WSN lifetime is nothing but Low Energy Adaptive Clustering Hierarchy (LEACH) and a flexible AODV-based (Ad hoc On-Demand Distance Vector) routing protocol $[14,15]$. LEACH acquaint with an algorithm for rotating $\mathrm{CHs}$ positions and adapts clusters to regularly allocate the energy load amid all SNs and thus allows WSNs self-organization.

The $\mathrm{CH}$ selection can be done by Hybridization of residual energy of a node and its degree or proximity to its neighbor is in the process of HEED (Hybrid EnergyEfficient Distributed clustering) [16]. HEED approach can be beneficial to design WSN protocols that need extended network lifespan, scalability, load balancing and fault tolerance but it delivers only algorithms for constructing a two-level hierarchy and there is no idea is offered for scheming a protocol to multilevel hierarchies. Power Efficient and Adaptive Clustering Hierarchy (PEACH) [17] chooses $\mathrm{CHs}$ which supports adaptive multilevel clustering and devoid of the added overhead of wireless communication for both location-aware and Location unaware WSNs but with low scalability and high latency which makes it only appropriate for small networks. Energy Efficient Multi-level Clustering (EEMC) [18] attains energy consumption lesser and least dormancy in WSNs by the formation of multi-level clustering with least algorithm above. Though, it disregards the problem of channel pileup that occurs often in wireless networks. Energy Efficient Heterogeneous Clustered scheme (EEHC) [19] chooses each node's weighted election probabilities based on $\mathrm{CHs}$ is a function of the residual energy and then further supports the node heterogeneity in WSNs. Energy efficient cross layer routing protocol for networks which are heterogeneous and depends on adaptive threshold sensitive distributed routing protocol for WSN. CHs are selected on the basis of node weighted probability [20]. Fuzzy logic based energy and dynamic clustering with Ant Colony Optimization (ACO) metaheuristic was based on Multi path Routing Protocol (MRP) $[21,22]$. $\mathrm{CH}$ is designated based on the remaining nodes energy and an enhanced ACO algorithm is implemented to hunt multiple paths which exist between $\mathrm{CH}$ and BS. Mobility-Based Clustering (MBC) protocol [23] supports mobility of node; therefore $\mathrm{CHs}$ will be chosen based on mobility and nodes residual energy, while a non- $\mathrm{CH}$ node upholds stability linkage with its $\mathrm{CH}$ throughout the set-up phase. Ray and De (2016) [24] proposed Efficient Energy Clustering Protocol based on Kmeans (EECPK - means) midpoint algorithm in WSNs. Their research work delivers an enduring process and 
greater expense of energy than our scheme necessities. A comparison of protocols in WSNs emphasizing their features and restrictions for an improved insight are represented in Table 1.

It is desperately known from the comparison that Computational Intelligence (CI) as well as classical based methods have their restrictions as well as personal features. Classical methods are improved in load balancing, selforganization with least above but normal in energyefficiency while CI based metaheuristic are exposed to be fine with energy- efficiency with extended life of network. Consequently, CI based metaheuristic methods must be more reconnoitered and enhanced for WSN's energyefficient solutions. The clustering techniques for different attributes are given in Table 2. Owing to goals, the needed clustering process and cluster properties are also mentioned in Table 1 and 2, it was found that compared with state-ofthe-art methods, PSO has general good clustering attributes.

Table 1. Comparison of protocols in WSNs.

\begin{tabular}{|c|c|c|c|c|c|}
\hline Protocol & Classification & $\begin{array}{l}\text { Energy- } \\
\text { efficiency }\end{array}$ & Features & Limitations & References \\
\hline EEHC & Classical & Good & Support node heterogeneity & Low scalability & [19] \\
\hline $\mathrm{MBC}$ & Classical & Average & $\begin{array}{l}\text { High node mobility, low } \\
\text { packet loss }\end{array}$ & High communication cost & {$[23]$} \\
\hline PSO & $\begin{array}{l}\text { Computational } \\
\text { Intelligence }\end{array}$ & Good & Better packet delivery & Network overhead & {$[25]$} \\
\hline HEED & Classical & Average & Low communication cost & High latency & [16] \\
\hline EECF & Classical & Average & Prolong network lifetime & Low transmission range & [9] \\
\hline ERP & $\begin{array}{l}\text { Computational } \\
\text { Intelligence }\end{array}$ & Average & Better network lifetime & $\begin{array}{l}\text { Non-cluster based } \\
\text { approach }\end{array}$ & [26] \\
\hline LEACH & Classical & Average & Self-organization & High communication cost & [14] \\
\hline EEMC & Classical & Average & $\begin{array}{l}\text { Minimum overhead, low } \\
\text { latency }\end{array}$ & $\begin{array}{l}\text { Only uniform node } \\
\text { distribution }\end{array}$ & {$[18]$} \\
\hline PEACH & Classical & Average & Load balancing & $\begin{array}{l}\text { High latency, low } \\
\text { scalability }\end{array}$ & {$[17]$} \\
\hline
\end{tabular}

Table 2. Different attributes of clustering techniques.

\begin{tabular}{|c|c|c|c|c|c|c|c|c|c|}
\hline \multicolumn{3}{|c|}{ Cluster properties } & \multicolumn{3}{|c|}{ CH capabilities } & \multicolumn{3}{|c|}{ Clustering process } & \multirow[t]{2}{*}{ Ref } \\
\hline Clustering approach & Cluster count & Stability & Mobility & Role & Node type & $\begin{array}{l}\text { Metho } \\
\text { dology }\end{array}$ & Method complexity & Objective & \\
\hline SEP & Variable & Provisioned & Stationary & Relaying & Heterogenous & Distributed & Constant & Save energy & [27] \\
\hline LEACH & Variable & Provisioned & Stationary & Relaying & Homogeneous & Distributed & Constant & Save energy & [14] \\
\hline AZR-LEACH & Pre-set & Fixed & Stationary & Relaying & Heterogenous & Distributed & Constant & Scaliblity & [28] \\
\hline HEER & Variable & Assumed & Stationary & Aggregation & Homogeneous & Distributed & Constant & Save energy & [29] \\
\hline EADC & Variable & Provisioned & Stationary & Aggregation & Homogeneous & Distributed & Variable & Balance energy & [30] \\
\hline DHCR & Variable & Provisioned & Stationary & Relaying & Homogeneous & Distributed & Variable & Load balancing & [31] \\
\hline
\end{tabular}

\section{Proposed Work}

An enormous range of clustering protocols has been established for WSNs. In the present study, GA based EECP were proposed recently to determine end to end delay, packet delivery ratio, energy consumption and throughput. Owing to the sensor nodes energy limit, extending wireless sensor networks (WSNs) lifetime became a very large challenging issue. To conquer this kind of problem, Efficient Energy Clustering Protocol (EECP) was proposed. These defy turn out to be even more critical in wide-ranging sensor networks where much more energy is consumed owing to further data groups and packet broadcasts. Clustering based protocols are supposed to be believed as the top selection for that kind of WSNs. The proposed EECP plays a vital role for the partition of a large-scale network into shaped clusters. Based on clustering scheme, dissimilar energy saving approaches is proposed, such as that of efficient $\mathrm{CH}$ relay selection and re clustering locality.

The proposed energy saving approaches was simpler but hotspot response and robust routing. Performance analysis demonstrated that proposed EECP can skillfully save energy that is far improved than the hybrid, distributed clustering and energy-efficient in terms of packet collision rate and energy saving in WSN environment. Planning energy efficient clustering technique is significant for extending the network lifetime. There are about three types of routing protocols namely, clustering protocols, direct communication and minimum transmission energy. In the direct communication, SNs straightly interconnect with BS. Though, more energy is needed for communication as the network size upsurges and then it causes SNs to die very rapidly.

\section{A. Design flow of WSN}

The data flow illustration of proposed EECP protocol is given in Fig. 2 which represents the formation of the cluster region and energy harvesting was described by the below important seven steps.

Step 1: The BS monitors WSNs mobile sensor nodes and it forms cluster region separately.

Step 2: Each cluster region collects the data from cluster nodes via Efficient Energy Saving Clustering Formation (EESCF) algorithm. This algorithm was used to credit each active node energy status when data's are transmitting and rest of the mobile sensor nodes are put into the sleep mode status in the situation. So, it can be easily identified all the initial level of mobile nodes which are put into the sleep mode then assign the initial energy to be monitored. 


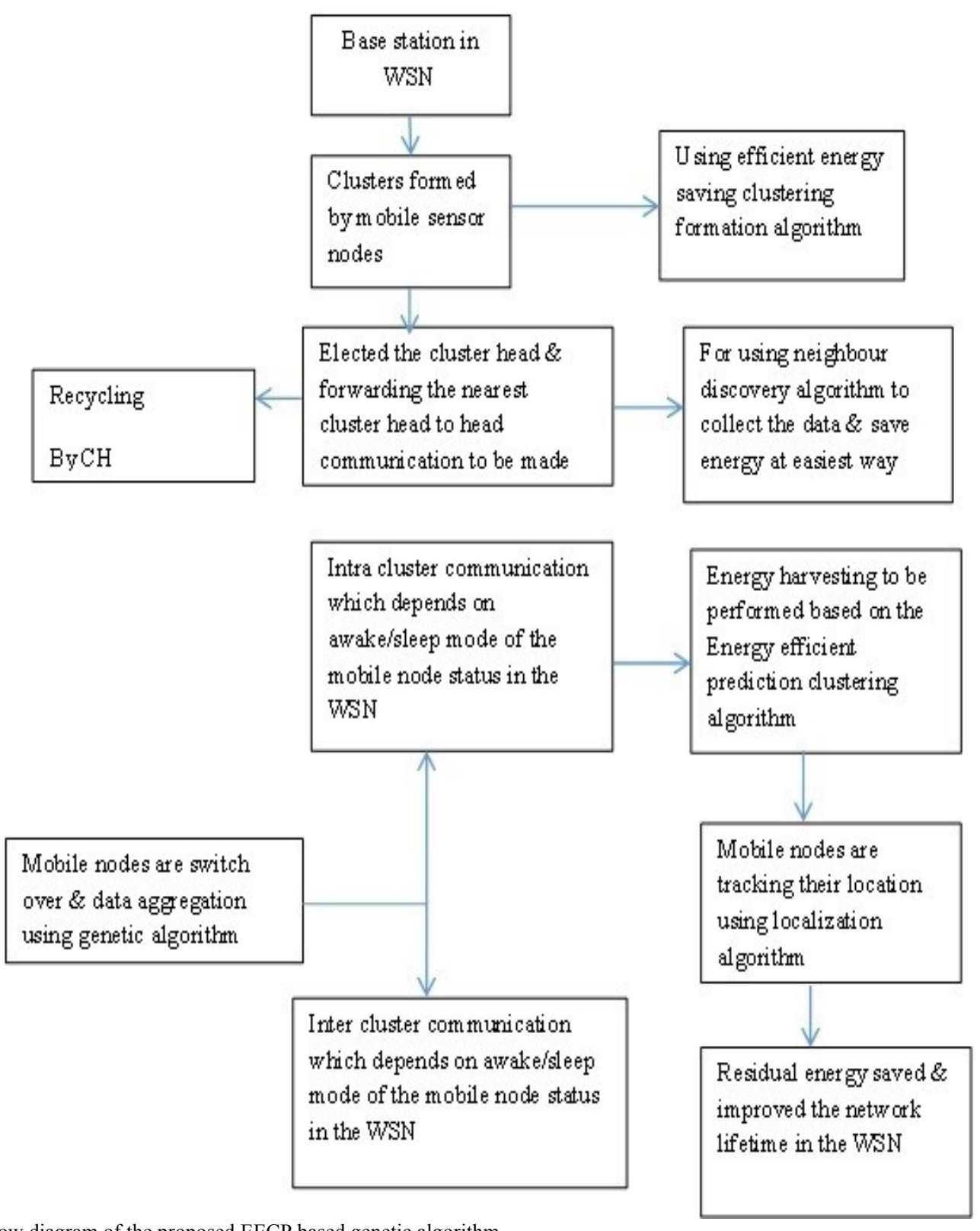

Fig. 2. Data flow diagram of the proposed EECP based genetic algorithm

Step 3: The CHs are elected from each group of cluster regions and head to head communication are formed using Neighbor Discovery Algorithm. This algorithm performs the nearest $\mathrm{CH}$ to be chosen and it supports to save the energy in the easiest way in the network communication. This should be maintained at each cycling of cluster nodes formation. When new mobile nodes are arrived or moved out then it detects the known mobile nodes based on this algorithm along with the cluster regions.

Step 4: After recycling each cluster groups and newly elected $\mathrm{CH}$ in each cluster region in the network, mobile nodes are switch over from one location to another location to aggregate the data in the network by using Genetic Algorithm.

Step 5: Each data aggregation using Genetic Algorithm identifies the node status level of intra cluster and inter cluster communication to be performed in network.

Step 6: Network harvesting the energy level for each cluster group and balancing the cluster nodes at uniformly identified at easiest way then they predict the node status which beyond to goes to dead cell situation to be dropped by using Energy Efficient Prediction Clustering Algorithm in the network.

Step 7: Mobile nodes will track their location by using Localization Algorithm for remaining energy saved and improved the network lifetime in the WSN.

\section{B. Network model}

The most significant issues concerns clustering are to develop cluster structure, diminish energy intake for data broadcast and optimize the choice of CHs. This inspires us to suggest Genetic Algorithm which is based on EECP. The foremost offerings of the proposed method are as below:

(i) Proposed EECP algorithm computes the optimal no of preferred clusters based on the sensing area size and sensors quantity present in it.

Optimum quantity of clusters $K_{o p}$ can be obtained as follows: 
$k_{o p}=\frac{\sqrt{S}}{\sqrt{2 \Pi}} \sqrt{\frac{\epsilon_{f s}}{\epsilon_{m p}}} \frac{N}{d_{B S}^{2}}$

Here $S$ is the entire sensor nodes amount equally dispersed in $N \times N$ sensing region, $d_{B S}$ is distance from $\mathrm{CH}$ to BS, $\in_{f s}$ is stricture for permitted space model and $\in_{m p}$ is stricture for multipath model.

(ii) To balance the energy consumed, BS can be replacing in the best location to communicate with $\mathrm{CH}$. The prime objective is to conclude the finest location of BS comparatively to different clusters moulded by considering that the BS has comparatively adequate energy [32]. To determine the optimization problem that minimizes the overall energy consume by lively sensors in the network as follows:

$\min g(p, q)=e_{0} \sum_{i \in Z}\left[\left(p_{i}^{o p}-p\right)^{2}+\left(q_{i}^{o p}-q\right)^{2}\right]^{\alpha / 2}$

Where $Z=S_{C H},\left(p_{i}^{o p}, q_{i}^{o p}\right)=$ input data.

(iii) Initially all the mobile nodes will have same energy level so the $\mathrm{CH}$ is elected by random process where $\mathrm{CHs}$ are equally dispersed and every cluster contain a nearly identical quantity of SNs. Accordingly, load of CHs turn out to be balanced, which eventually extend network lifespan.

(iv) The consumption of energy for $n$ bits of data transmission consists about three parts [33] the energy used up by the transmitter $\mathrm{E}_{\mathrm{tr}}$, by the receiver $E_{r e}$ and by acknowledgement (ACK) packet exchange $E_{a k}$

$$
E_{\text {total }}(n, m)=E_{t r}(n, m)+E_{r e}(n, m)+E_{a k}
$$

Currently, for transmitting $n$ bits of data, energy consumption is represented by

$$
E_{t r}(n, m)=n \cdot E_{\text {ele }}+E_{a p}(n, m)
$$

Additionally, if the distance between receiver and transmitter is $m$, then

$$
E_{t r}(n, m)=\left\{\begin{array}{l}
n E_{\text {ele }}+n \in_{f s} m^{2} \text { if } m<m_{0}, \\
n E_{\text {ele }}+n \in_{m p} m^{4} \text { if } m \geq m_{0}
\end{array}\right.
$$

Where $m_{0}$ is threshold distance and also receive $n$ bit message, the radio spends $E_{r e}(n, m)$ as follows

$$
E_{r e}(n, m)=n \cdot E_{\text {ele }}
$$

For ACK packet exchange energy consumption is stated as

$$
E_{a c k}=\tau_{a c k}\left(E_{t r}+E_{r e}\right)
$$

Where $\tau_{\text {ack }}=n_{\text {ack }} / n$ is the ratio between lengths of data packet to acknowledgement the packet. The transmitter consumes energy to run radio electronics and power amplifier. The receiver also consumes energy to run radio electronics. When a node sends message of $M$ bits through a distance of $d$, the node expends $E_{T x}(M, d)$ energy:

$$
E_{T X}(M, d)=\left\{\begin{array}{l}
M E_{\text {elec }}+M \varepsilon_{f_{s}} d^{2} d \leq d_{0} \\
M E_{\text {elec }}+M \varepsilon_{m p} d^{4} d>d_{0}
\end{array}\right.
$$

When the node receives this message, it expends $E_{R x}(M)$ energy as:

$$
E_{R x}(M)=M E_{\text {elec }}
$$

Where $\varepsilon_{f s} d^{2},\left(\varepsilon_{\text {amp }} d^{4}\right)$, and $E_{\text {elec }}$ are the amplifier energy and electronics energy, respectively.

(v) Inter-cluster multi-hop data routing Initially, all network SNs are arranged hierarchically to build clusters. Since, the nodes are formed by first ring are used to communicate directly with BS, they will not participate in clustering technique. $\mathrm{CH}$ from $n^{\text {th }}$ ring receives data traffic from its cluster members in addition to relaying data traffic from $\mathrm{CHs}$ in $(n+1)^{\text {th }}$ ring and communicates the mutual traffic to $\mathrm{CH}$ in $(n-1)^{\text {th }}$ ring. Data forwarding lasts hop-byhop till it reaches destination node. Every $\mathrm{CH}$ forwards its information to the BS via descending cluster heads in the lower levels. This promotes minimum hop routing with at most $n$ hops in between source $\mathrm{CH}$ and $\mathrm{BS}$ if $\mathrm{CH}$ from $n^{\text {th }}$ level.

(vi) $\mathrm{CH}$ selection phase In the beginning of the process with some probability $K$, huge numbers of Tentative $\mathrm{CHs}$ (T-CHs) are chosen from each level [34]. Where, $K$ varies dynamically since percentage of $\mathrm{CHs}$ differs from level to level with threshold $T$ is being application specific. Equation (10) represents a probability value $K_{M n}$ for a given level number $M_{n}$. Non-competing nodes goes into sleep mode, when $\mathrm{T}-\mathrm{CH}$ s compete each other for final $\mathrm{CH}$ s role.

$$
K_{M n}=1-\frac{M_{n}}{M_{t}} \forall i>1
$$

Where $M_{n}$ is the line number and $M_{t}$ is total number of levels in the network.

(vii) Cluster Formation Phase When the entire final $\mathrm{CHs}$ are designated, each $\mathrm{CH}$ broadcasts a message, $C H-A D V$ $-M S G$. The non-competing sleeping nodes wake-up and join nearby $\mathrm{CH}$ which has high RSSI by transmitting $J O I N-C H-M S G$ message.

\section{Proposed EECP-Genetic Algorithm}

The $\mathrm{CH}$ gathers the data from the mobile nodes and stores for future transmission or reception purpose. Once combining the data, $\mathrm{CH}$ directs a sleep notification to the mobile sensor node and drives to the sleep mode based on energy level with repetition of data occurred. Mobile Sensor Node sends wake up signal to the $\mathrm{CH}$ gathers whereas interactive $\mathrm{CH}$ attains wake state and then it uploads the data to mobile sensor node. If the two Cluster Head directs notification at same time, then the mobile sensor node can collect data from both $\mathrm{CH}$ instantaneously.

Mobile Sensor Node data energetically used genetic scheme. Initially, mobile sensor node data sends to $\mathrm{CH}$ gathers and verifying the repeated message which has lesser energy level and then changes to another $\mathrm{CH}$ (inter cluster) communication. If the $\mathrm{CH}$ has any of buffer overflow issue and power issue then it will send a caution 
message to the mobile sensor node for the collection of data instantly. For uploading data to change $\mathrm{CH}$, mobile sensor node collects data instantly and returns to the BS.

If $\mathrm{CH}$ was departed due to power drain, then higher energy node inside the cluster (intra cluster) will be selected as new $\mathrm{CH}$, which acts as previous $\mathrm{CH}$ role. Mobile sensor node once upload the data to the BS goes to sleep state in terms of decreasing the energy wastage. Another mobile sensor node will come into play if power drains.

\section{Pseudo code for Genetic Algorithm}

Genetic Algorithm (Z) -> (A, B)

1. FOR $\mathrm{j}<-1$ TO ZDO

2. A $<-$ Random Node Selection $(Z)$;

3. $\mathrm{B}<$ - Optimal Partition (Z,A);

4. Sort Cluster Range(A,B);

5. REPEAT

6. $\{\mathrm{A}, \mathrm{B}\}<-$ Create New Connections $(\{\mathrm{A}, \mathrm{B}\})$;

7. Sort Cluster Range (A,B);

8. Create New Connections $(\{\mathrm{A}, \mathrm{B}\})->\{\mathrm{A}($ new $)$, $\mathrm{B}($ new) $\}$;

9. A (new-1), B (new-1) <- A(1), B(1);

10. FOR $\mathrm{j}<-2$ TO ZDO

11. $(\mathrm{x}, \mathrm{y})<-$ Select Next Pair;

12. A (new-1), B(new-1) <- Cross Cluster

Communica- tion( $\mathrm{A}(0), \mathrm{B}(0), \mathrm{A}(1), \mathrm{B}(1))$;

13. Iterate $\mathrm{K}-\mathrm{Means}(\mathrm{A}($ new-1), $\mathrm{B}($ new-1);

14. Cross Cluster

Communication(A(1),B(1),A(2),B(2)) -> (A(new),B(new)

15. $\mathrm{A}$ (new) <- Combine Centroids (A (1), A(2));

16. $\mathrm{B}($ new) <- Combine Partitions (B (1), B(2));

17. A (new) <- Update Centroids(A (new), B (new));

18. Remove Empty Clusters(A (new), B(new));

19. IS(A (new), B (new);

20. Combine Centroids (A (1), A (2)) ->A

(new); 21. A (new) <- A (1) U A (2)

22. Combine Partitions (A (new), B (1), B (2)) -> B (new)

23. FOR $\mathrm{i}<-1$ TO NDO

24. IF $\|\mathrm{z}-\mathrm{c}\| 2<\|\mathrm{z}-\mathrm{c}\| 2$ THEN

25. $\mathrm{B}<-\mathrm{B}(1)$

26. ELSE

27. $\mathrm{B}<-\mathrm{B}(2)$

28. END-FOR

29. Update Centroids (A (1), A (2)) ->A (new)

30. FOR $\mathrm{j}<-1 \mathrm{TO} \mid \mathrm{A}($ new $) \mid \mathrm{DO}$

31. Aj (new) <- Calculate Centroid (B (new), j);

32. FINISHED

\section{Simulation Parameters}

This paper assumes 100 (i.e. $n=100$ ) mobile sensor nodes that are arbitrarily deployed inside the area of $100 \mathrm{~m} \times 100 \mathrm{~m}$. The three kinds of nodes may include normal nodes, advance nodes, and super nodes with the initial energy of $0.25 \mathrm{~J}, 0.5 \mathrm{~J}$ and $0.75 \mathrm{~J}$ respectively. The BS was located at any random place outdoor the sensing field. The remaining all parameters are mentioned in Table III. The SNs sense the data at a fixed rate and these SNs always have some data to send. This work also undertakes a simple radio energy model as conversed in Sabet and Naji [31].
Table 3. Simulation

Parameters

\begin{tabular}{l|l}
\hline \multicolumn{1}{c|}{ Parameter } & \multicolumn{1}{c}{ Value } \\
\hline $\begin{array}{l}\text { Number of Cluster } \\
\text { Head }\end{array}$ & 5 \\
$\begin{array}{l}\text { Nodes of Mobile } \\
\text { Number of Base } \\
\text { Station }\end{array}$ & 1 \\
Network Size & \\
Protocol Usage & 2000 X 1100 \\
& Efficient Energy \\
Algorithm Design & Clustering Protocol \\
& EESCF, Neighbor \\
& Discovery, EECP, \\
& Genetic, \\
Range of cluster & Localization \\
formation & $500 \mathrm{~m}(300 \mathrm{~m}-$ \\
Throughput & $600 \mathrm{~m})$ \\
Bandwidth & $50 \mathrm{Mbps}(9.0$ \\
Frequency & Mbps, 20 Mbps) \\
Packet Transmission & $400 \mathrm{Mbps}$ \\
Packet Rate & $50 \mathrm{~Hz}$ \\
Request message & $1000 \mathrm{Bytes}$ \\
interval & \\
Simulation Time & $250 \mathrm{Packets} \mathrm{per}$ \\
\hline
\end{tabular}

\section{Results and Discussion}

The results of proposed EECP has evaluated and compared the results with existed LEACH, PSO HSCAP by using MATLAB simulator. The simulation results give the parameters energy efficiency, PDR (packet delivery ratio), throughput and average latency. The simulation has performed by using EECP genetic algorithm and free space propagation. In WSN scenario, a SNs network extending from 20 to 100 is installed arbitrarily above an area of size $2000 \times 1100$ with a BS placed at $(300 \mathrm{~m}, 600 \mathrm{~m})$ within the network field. Table IV displays the evaluation of proposed EECP genetic algorithm with EECP-K means algorithm, Hybrid algorithm, Neighborhood algorithm and Greedy algorithm in WSNs.

1) Network throughput: The amount of packets received by the BS from non-base station nodes is called throughput. As number of nodes increases throughput will increase in all other data aggregation and EECP genetic algorithm data aggregation provides throughput as 95 Mbps. The proposed EECP-GA provides maximum throughput when compared to existing EECP-K means algorithm. Fig. 3 defines the throughput evaluation amongst EECP genetic algorithm and its contestant's. Throughput of EECP genetic algorithm as 95, 90, 80, 74 and 67 Mbps was compared greater than EECP-K means algorithm, Hybrid algorithm, Neighborhood algorithm and Greedy algorithm respectively. The throughput of proactive routing protocol is openly linked to cluster lifetime, higher cluster life time effects higher throughput. EECP Genetic Algorithm life time is superior to its opponents, then it has superior throughput that is evidently stated in Fig. 3.

2) Energy consumption: Energy used by the network is proportional to the diameter of the network. By using SEED algorithm, network energy was found to be 
increased from $100 \mathrm{~nJ} / \mathrm{bit}$ to $200 \mathrm{~nJ} / \mathrm{bit}$ when network size was increased from $100 \mathrm{~m} \times 100 \mathrm{~m}$ to $200 \mathrm{~m} \times 200 \mathrm{~m}$. Network size, topology changes and energy intake are the factors which don't effect on protocols duties and their behavior in energy efficient protocols [35]. Figure 3: Comparison of throughput Fig. 4 depicts the energy consumption between proposed EECP based Genetic Algorithm and its competitors. Energy consumption of EECP-GA as 99, 95, 80, 68 and 56 was compared greater than EECP-K means algorithm, Hybrid algorithm, Neighborhood algorithm and Greedy algorithm respectively. The behavior of EECP Genetic Algorithm remains consistent from $300 \mathrm{~m} \times 300 \mathrm{~m}$ to $500 \mathrm{~m} \times 500$ $\mathrm{m}$. Additional upsurge of network size and energy consumption level was augmented which was obviously specified in Fig. 4. Nevertheless, the proposed model's energy consumption residues equal with cumulative size of the sensing field.

3) Cluster formation: The cluster formation phase during every single node which is acknowledged by $\mathrm{CH}$ $\mathrm{Msg}$ from neighbors and its distance with the $\mathrm{CH}$ is fewer than with intra-cluster radio radius $R C$, chosen the utmost suitable $\mathrm{CH}$ with an expedient and operative method. $\mathrm{CH}$ alone positioned in the verge of the tree is formed when a node had not accepted any $\mathrm{CHs}$ statement. Though, a node accepted $\mathrm{CH}-\mathrm{Msg}$ from just one adequate $\mathrm{CH}$ will join to that $\mathrm{CH}$ with propagating Join-Msg. As number of nodes increases cluster formation will also increase in all other data aggregation and the EECP-GA data aggregation provides maximum number of cluster formation as 97 was high compared to its competitors. Initially, $\mathrm{CHs}$ set are stated gradually from layer near to BS to whole network. The CEBCRA protocol $\mathrm{CH}$ directs data to BS, it chooses a best nominee among neighbors first and it allow itself for maximum price of path [36]. If, a best nominee is $\mathrm{CH}$, then the data has been directed to BS or else, the data has been frontwards to best nominee node which are selected. Cluster formation of EECP genetic algorithm as 97, 94, 90, 83 and 75 was compared greater than EECP-K means algorithm, Hybrid algorithm, Neighborhood algorithm and Greedy algorithm respectively. The behavior of EECPGA remains that node numbers upsurge the network size the cluster formation is also augmented which is evidently revealed in Fig. 5.

4) Packet Delivery Ratio: Packet delivery ratio is known as the data packet's ratio acknowledged by the terminus to those made by the source and it may be known as the ratio of $\mathrm{d} 1$ and $\mathrm{d} 2$, where, each endpoint receives data packets whose amount is denoted by $\mathrm{d} 1$ and each source made data pack- ets whose amount is denoted by d2. Fig. 6 display that the comparison of packet delivery ratio between proposed EECP- genetic algorithm and its competitors. As the integer of nodes upsurges the packet delivery ratio will increase in all other data aggregation and the EECP-GA data aggregation provides maximum packet delivery ratio as $99 \%, 98 \%$, $90 \%, 88 \%$ and $85 \%$ was compared higher than EECP-K means algorithm, Hybrid algorithm, Neighborhood algorithm and Greedy algorithm respectively for 100 nodes and supply uppermost quantity of packets amid it's all peers, even at uppermost node densities. Beecluster supply nearly $98 \%$ packets at 100 nodes with BS located at optimal position in WSN. EECP-Genetic Algorithm has uppermost PDR when compared to Beecluster,
BeeSensor, MRP and ERP at nodes extending from 100 to 700 [33].

5)End-to-End Delay: End-to-End Delay denotes the time engaged for a packet to be transferred through a network from origin to endpoint. It is a general word in IP network monitoring and varies from Round-Trip Time (RTT). As the integer of nodes upsurges the end to end delay will decrease in all other data transmission and the EECP genetic algorithm data transmission provides maximum end to end delay as $30 \mathrm{~ms}, 36 \mathrm{~ms}, 37 \mathrm{~ms}, 38 \mathrm{~ms}$ and $40 \mathrm{~ms}$ was compared lower than EECP-K means algorithm, Hybrid algorithm, Neighborhood algorithm and Greedy algorithm respectively for 100 nodes. Fig. 7 illustrates the end to end delay time of sensing area was found to be $500 \mathrm{~m} \times 500 \mathrm{~m}$. The end to end delay time of proposed EECP-GA scheme is lower than that of PEGASIS scheme [37].

Fig. 8 shows an analysis of different parameters of EECP genetic algorithm; EECP-K means algorithm, Hybrid algorithm, Neighborhood algorithm and Greedy algorithm. EECP-genetic algorithm based clustering algorithm consumes less energy, high throughput, high packet ratio with minimum end to end delay while conferred with other procedures and prolong network life.

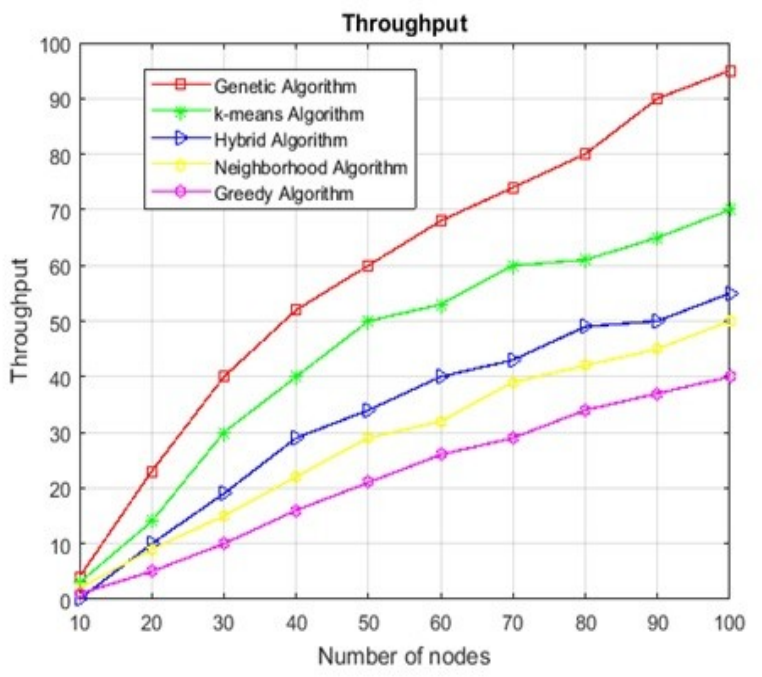

Fig. 3. Comparison of throughput

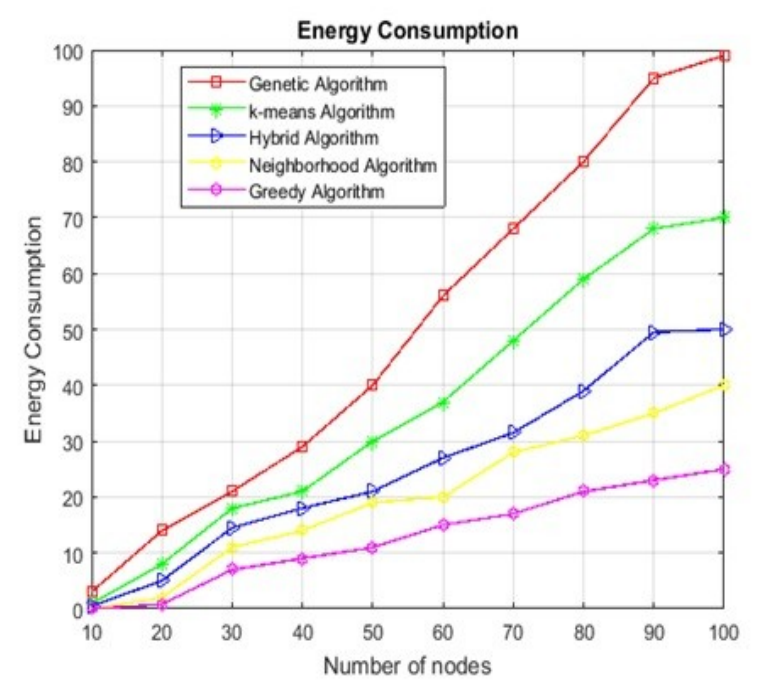

Fig 4. Comparison of energy consumption 


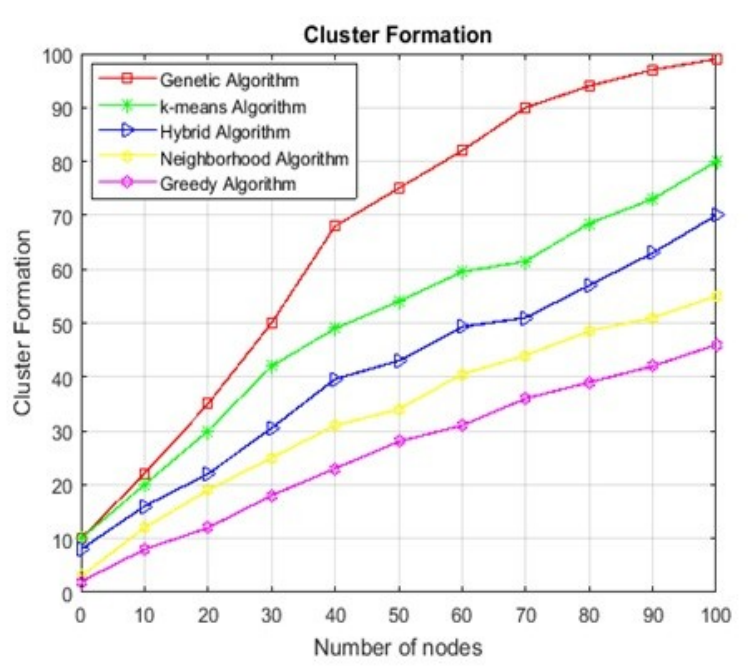

Fig. 5. Comparison of cluster formation

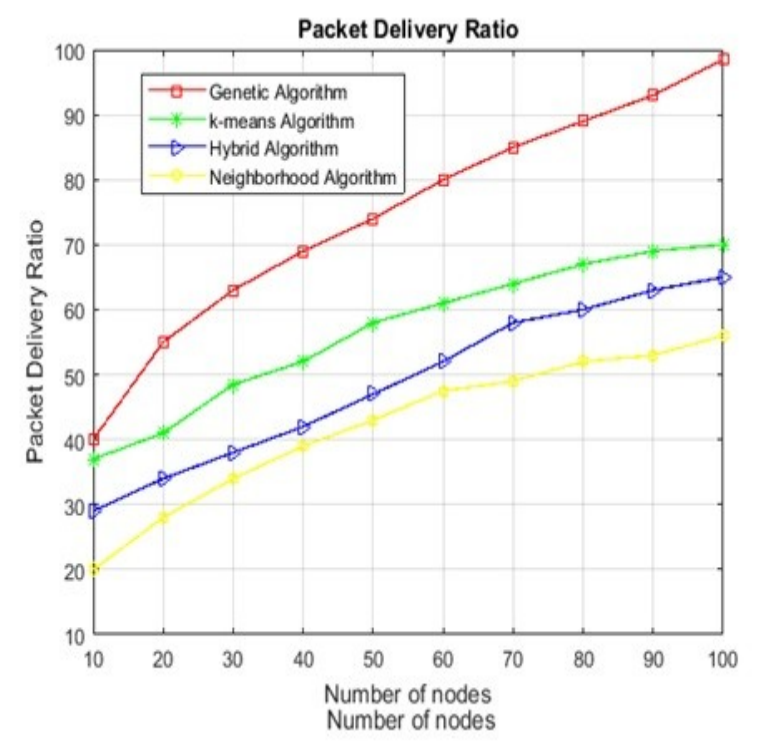

Fig. 6. Comparison of packet delivery ratio

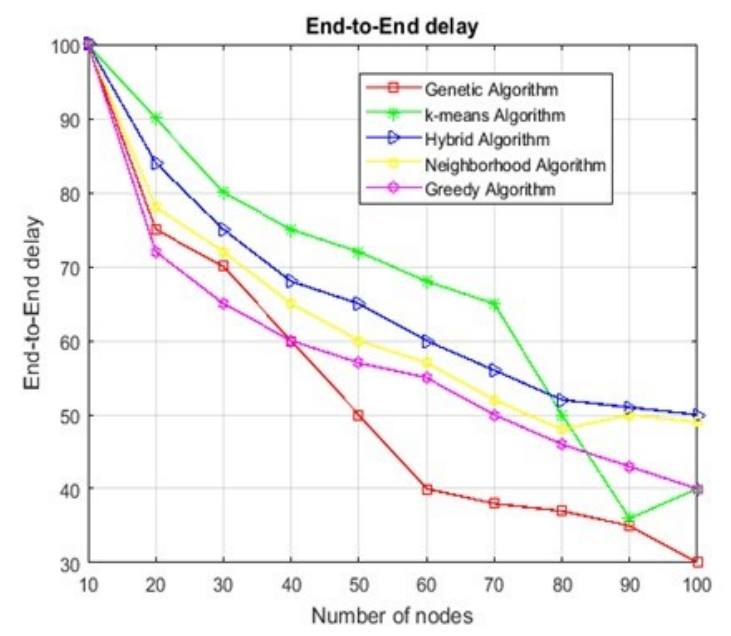

Fig. 7. Comparison of End to End dela

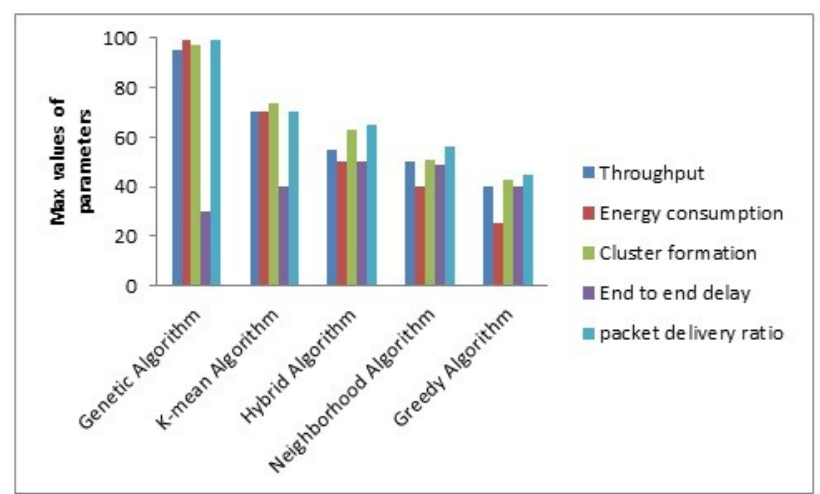

Fig. 8. Analysis of different parameters of Genetic Algorithm, K-mean Algorithm, Hybrid Algorithm, Neighborhood Algorithm and Greedy Algorithm

\section{Conclusion}

Ideal use of resources in the network is one among the utmost significant subjects in sensor networks. Normally replacement of energy supply and reprocessing of a node is not affordable or surroundings where there is no likelihood of exchanging in these networks. Hence, saving energy consumption in each node will considerably diminish the cost of network conservation and upsurges lifespan of the network. Cluster-based routing scheme is a well-organized technique which utilizes less energy through aggregation and data combination to decrease the quantity of messages directed to central station. The present study states EECPGA, a clustering procedure for WSNs based on metaheuristic which is used by $\mathrm{CH}$ and cluster members for improved solution of search equation. The proposed EECP protocol with genetic algorithm utilizes an energyefficient method, which picks optimal $\mathrm{CHs}$ based on an enhanced search equation with effective fitness function to improve exploitation competences in addition to convergence rate of existing metaheuristic. The evaluation of the proposed procedure's performance with famous other cluster based procedures was done to show its validness above numerous metrics of presentation. Simulation results showed that EECP-genetic algorithm based clustering consumes fewer energy while comparing with other procedures and extend life of network although supplying uppermost quantity of packets with least end-to end delay in varied WSNs circumstances. Energy consumption of network is greatly reduced by EECPGenetic algorithm as compared with the various algorithms. In WSN during the data exchanging times, whenever a new cluster is formed some nodes are getting congested paths from or to the neighbor nodes or $\mathrm{CH}$ nodes. During this congestion time, network draws more energy to deliver packets. In future work, this congestion will try to avoid in the network and also taking into consideration on energy harvesting scheme to increase the network life time.

This is an Open Access article distributed under the terms of the Creative Commons Attribution License

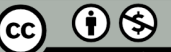




\section{References}

[1] Lo C, Lynch JP, Liu M. "Distributed model based nonlinear sensor fault diagnosis in wireless sensor networks". Mechanical System Signal Process, 66, p. 470-484, (2016).

[2] Delamo M, Felici-Castell S, Perez-Solano J.J, Foster A. "Designing an open source maintenance-free environmental monitoring aplication for wireless sensor networks". Journal of System Software, 103, p. 238-247, (2015).

[3] Wang JC, Lin CH, Siahaan E, Chen BW, Chuang HL. "Mixed sound event verification on wireless sensor network for home automation". IEEE Trans Ind Information, 10(1), p. 802-812, (2014).

[4] Dima SM, Panagiotou C, Tsitsipis D, Antonopoulos C, Gialelis J, Koubias S. "Performance evaluation of a WSN system for distributed event detection using fuzzy logic". Ad Hoc Networks, 23, p. 87-108, (2014).

[5] Di Stefano A, La Corte A, Leotta M, Lio P, Scata M. "An IoTs algorithm in WSNs based on heuristics behavior and clustering methods". Ad Hoc Networks, 11(8), p. 2637-2647, (2013).

[6] Arunraja M, Malathi V, Sakthivel E. "Energy conservation in WSN through multilevel data reduction scheme". "Microprocess and Microsystems", 39(6), p. 348-357, (2015).

[7] Tyagi S, Kumar N. "A systematic review on clustering and routing techniques based upon leach protocol for wireless sensor networks". Journal of Network and Computer Aplications, 36(2), p. 623-645, (2013).

[8] Abbasi A, Younis M. "A survey on clustering algorithms for wireless sensor networks". Computer Communications, 30(14), p. 2826-2841, (2007).

[9] Chamam A, Pierre S. "A distributed energy-efficient clustering protocol for wireless sensor networks". Computer Electrical Engineering, 36(2), p. 303-312, (2010).

[10] Yahiaoui S, Omar M, Bouabdallah A, Natalizio E, Challal Y. "An energy efficient and QOS aware routing protocol for wireless sensor and actuator networks". AEU- International Journal of Electronics and Communications, 83, p.193-203, (2018).

[11]Khalil A, Bara A. "Energy-aware evolutionary routing protocol for dynamic clustering of wireless sensor networks" Swarm Evolution Computers, 1(4), p. 195-203, (2011).

[12] Kulkarni RV, Forster A, Venayagamoorthy GK. "Computational intelligence in wireless sensor networks: a survey". IEEE Communications, 13(1), p. 68-96, (2011).

[13] Srikanth. N, Siva ganga Prasad. M, "Efficient Clustering Protocol Using Fuzzy K-means and Midpoint Algorithm for Lifetime Improvement in WSNs", International Journal of Intelligent Engineering Systems, 11(4), p. 61-71, (2018).

[14]Heinzelman WB, Chandrakasan AP, Balakrishnan H. "An aplication specific protocol architecture for wireless microsensor networks". IEEE Trans Wireless Communications, 1(4), p. 660$670,(2002)$.

[15]T. Senthil and B. Kannapiran "ECTTM - Energy Conserving Trustworthy Topology Management Mechanism for Wireless Sensor Networks", International Journal of Intelligent Engineering and Systems, 10(4), p. 157-165,(2017).

[16] Younis O, Fahmy S. HEED: 'A hybrid, energy-efficient, distributed clustering aproach for ad hoc sensor networks". IEEE Transactions on Mobile Computing, 3(4), p. 366-379, (2004).

[17] Yi S, Heo J, Cho Y, Hong J. "PEACH: Power-efficient and adaptive clustering hierarchy protocol for wireless sensor networks". Computer Communications, 30(14), p. 2842-2852, (2007).

[18]Jin Y, Wang L, Kim Y, Yang X. "EEMC: An energy-efficient multi-level clustering algorithm for large-scale wireless sensor networks". Comput Networks, 52(3), p. 542-562, (2008).
[19]Kumar D, Aseri TC, Patel RB. "EEHC: Energy efficient heterogeneous clustered scheme for wireless sensor networks". Computer Communications, 32(4), p. 662-667. (2009).

[20]M. Tong and M. Tang, "LEACH-B: An improved LEACH protocol for wireless sensor network", In: Proc. of 6th International Conf. on Wireless Communications Networking and Mobile Computing, p. 1-4, 2010

[21] Yang J, Xu M, Zhao W, Xu B. "A multipath routing protocol based on clustering and ant colony optimization for wireless sensor networks". Sensors, 10(5), p. 4521-4540, (2010).

[22] Qadri MY, Qadri NN, McDonald-Maier KD. "Fuzzy logic based energy and throughput aware design space exploration for MPSoCs". Microprocess Microsystems, 40, p. 113-123, (2016).

[23]Deng S, Li J, Shen L. "Mobility-based clustering protocol for wireless sensor networks with mobile nodes". IET Wireless Sensor Systems, 1(1), p. 39-47, (2011).

[24]Amanpreet Kaur, Padam Kumar, Govind P Gupta, "Analysis on DV-Hop Algorithm and its variants by considering threshold" Journal of Telecommunication Electronic and Computers, 9(4), p. 79-83. (2017).

[25] Hradesh Kumar, Pradeep Kumar Singh, "Node Energy Based Aproach to Improve Network Lifetime and Throughput in Wireless Sensor Networks". Journal of Telecommunication Electronic and Computers, 9(3), p. 83-88, (2017).

[26] Srikanth. N, Siva ganga Prasad. M, "Energy efficient enhanced tree structured compression model (ET-CM) for data aggregation in wireless sensor networks", International Journal of Engineering and Technology, 7(2), p. 1-4, (2018).

[27] Bara'a AA, Khalil EA. "A new evolutionary based routing protocol for clustered heterogeneous wireless sensor networks". Aplied Soft Computing, 12(7), p. 1950-1957, (2012).

[28] Gurrutxaga I, Albisua I, Arbelaitz O, Martin JI, Muguerza J, Perez JM, Perona I. SEP/COP: "An efficient method to find the best partition in hierarchical clustering based on a new cluster validity index". Pattern Recognition, 43(10), p. 3364-3373, (2010).

[29]Khan ZA, Sampalli S. AZR-LEACH "An energy efficient routing protocol for wireless sensor networks". International Journal of Communication Networks System Sciences, 5(11), p. 785-789, (2012).

[30] Yi D, Yang H. HEER-“A delay-aware and energy-efficient routing protocol for wireless sensor networks". Computer Networks, 104, p. $155-173,(2016)$

[31]Yu J, Qi Y, Wang G, Gu X. “A cluster-based routing protocol for wireless sensor networks with non-uniform node distribution". AEU-International Journal of Electronics and Communications, 66(1), 2012.

[32] Sabet M, Naji HR. "A decentralized energy efficient hierarchical cluster-based routing algorithm for wireless sensor networks". AEU-International Journal of Electronics and Communications, 69(5), p. 790-799, 2015.

[33] Singh.D, Naik. S. K, "Enhanced modified LEACH (EMODLEACH) protocol for WSN", In Advanced Computing and Communication (ISACC), 2015 International Symposium on Advanced Computing and Communication, pp. 328-333. IEEE 2015.

[34] Singh.D, Binod Kumar Pattanayak, Chandan Kumar Panda "Analysis of an Improved Energy Balanced Routing Protocol for Wireless Sensor Network", IEEE International conference on Communication and Signal processing (ICCSP2016), p-1503-1507,April 2016.

[35] Singh.D, Binod Kumar Pattanayak "Analytical Study of an Improved Cluster based Routing Protocol in Wireless Sensor Network", Indian Journal of Science and Technology, Vol 9(37),p 1-8, October 2016. 